\title{
On the energy dependence of the steric effect in atom-molecule reactive scattering. 1. A quasiclassical approach
}

\author{
Gerrit C. Groenenboom and Anthony J. H. M. Meijer \\ Institute of Theoretical Chemistry, University of Nijmegen, Toernooiveld, 6525 ED Nijmegen, \\ The Netherlands
}

(Received 3 June 1994; accepted 28 July 1994)

\begin{abstract}
Experimental studies have shown that the steric effect in chemical reactions can decrease (e.g., for $\left.\mathrm{Ba}+\mathrm{N}_{2} \mathrm{O} \rightarrow \mathrm{BaO}^{*}+\mathrm{N}_{2}\right)$ or increase [e.g., for $\mathrm{Ca}\left({ }^{1} D_{2}\right)+\mathrm{CH}_{3} \mathrm{~F} \rightarrow \mathrm{CaF}^{*}+\mathrm{CH}_{3}$ ] with increasing translational energy. Decreasing (negative) energy dependences have successfully been modeled with the angle dependent line of centers model. We present a classical model in which a positive energy dependence of the steric effect is explained by an isotropic, attractive long range potential. In this "trapping" model we assume the reaction-apart from a cone of nonreaction at one side of the molecule- to be barrierless. This model shows that a positive energy dependence of the steric effect is not indicative of reorientation of the molecule, as has been suggested in the literature. Rather, the positive or negative energy dependence of the steric effect is shown to correlate with the absence or presence of a barrier to reaction and an attractive or repulsive long range potential. For the reorientation effects which occur in the case of anisotropic potentials, we consider the application of the standard quasiclassical trajectory (QCT) method and we introduce a modified QCT method. We argue that the latter is more suitable for the computation of the orientation dependent reactive cross section.
\end{abstract}

\section{INTRODUCTION}

The dependence of the reactivity on the orientation of the reagents is a key issue in dynamical stereochemistry. ${ }^{1,2}$ Experimentally, a symmetric top molecule with nonzero dipole moment (or a symmetric top like molecule such as $\mathrm{N}_{2} \mathrm{O}$ ) can be oriented using a hexapole state selector ${ }^{3}$ followed by a homogeneous electric field. This technique allows the control of the (average) orientation ( $\vartheta$ ) of the molecular symmetry axis with respect to the initial relative velocity of the reagents in a crossed beam experiment. The first experiments of this type were done by Brooks et al. ${ }^{4}$ and Beuhler et al. ${ }^{5}$ for the reactions of $\mathrm{K}$ and $\mathrm{Rb}$ with partially oriented $\mathrm{CH}_{3} \mathrm{I}$. Recently, Janssen, Parker, and Stolte ${ }^{6}$. performed experiments with well defined initial states for the reaction of $\mathrm{Ca}\left({ }^{1} D_{2}\right)+\mathrm{CH}_{3} \mathrm{~F}(J K M)$. They report the steric effect, i.e., the difference between the reactive cross sections for favorably and unfavorably oriented molecules relative to the reactive cross section for unoriented molecules, as a function of the relative translational energy for the $\left(v_{3}=0 ; J K M=111\right)$, $\left(v_{3}=0 ; J K M=212\right)$, and $\left(v_{3}=1 ; J K M=111\right)$ states (the $\nu_{3}$ vibrational mode is essentially a $\mathrm{C}-\mathrm{F}$ stretch vibration, $J, K$, and $M$ are the symmetric top quantum numbers for $\mathrm{CH}_{3} \mathrm{~F}$ ).

Most theoretical studies on orientational effects employ some version of the angle dependent line of centers model $(\mathrm{ADLCM})^{7}$ originally introduced by $\mathrm{Smith}^{8}$ and Pollak and Wyatt. ${ }^{9}$ This is a classical model in which the molecule is surrounded by an imaginary shell (usually a sphere) and it is assumed that a trajectory will be reactive if the radial kinetic energy at this shell is high enough to cross a barrier. This barrier is chosen to depend on the angle of attack $(\gamma)$ between the symmetry axis of the molecule and the line of centers (i.e., the line connecting the centers of mass of the two reactants). Usually, $\gamma=0^{\circ}$ corresponds to the relative orientation most favorable for reaction. Furthermore, the barrier is often taken to be infinite between a certain cutoff angle $\left(\gamma=\gamma_{c}\right)$ and $\gamma=180^{\circ}$. This region is called "cone of nonreaction."

The reason for the current study is the surprising positive energy dependence of the steric effect measured for the $\mathrm{Ca}\left({ }^{1} D_{2}\right)+\mathrm{CH}_{3} \mathrm{~F}$ reaction. With the ADLC model in mind this is counterintuitive; one would expect that at higher energies the trajectories will have enough energy to cross the angledependent barrier over a wider range of angles of attack $\gamma$, thus opening up the "cone of reaction" and lowering the steric effect. The ADLC model has successfully been used to account for the negative energy dependence of the steric effect in the reaction of $\mathrm{Ba}+\mathrm{N}_{2} \mathrm{O} .^{10}$

It has been suggested ${ }^{6,11}$ that the decrease of the steric effect at lower energies for the $\mathrm{Ca}\left({ }^{1} D_{2}\right)+\mathrm{CH}_{3} \mathrm{~F}$ reaction might be caused by reorientation of the $\mathrm{CH}_{3} \mathrm{~F}$ molecule due to anisotropic long range interactions between $\mathrm{CH}_{3} \mathrm{~F}$ and the electronically excited $\mathrm{Ca}\left({ }^{1} D_{2}\right)$. Supposedly, the " $\mathrm{F}$ end" of the $\mathrm{CH}_{3} \mathrm{~F}$ molecule would rotate towards the approaching $\mathrm{Ca}\left({ }^{1} \mathrm{D}_{2}\right)$, thus washing out the effect of the initial orientation of the molecule. At higher translational energies there would not be enough time for this reorientation to occur and the steric effect would increase.

However, in a series of trajectory calculations, employing several potential energy surfaces (PESs) (both ad hoc potentials and potentials based on computed electrostatic long range interactions) we found that the anisotropy in the potential, even though it can cause some reorientation, contributes little to the decrease of the steric effect. ${ }^{12}$ Under certain conditions, it might even increase the steric effect. At the same time, however, we find that it is possible to reproduce the experimentally found positive energy dependence of the steric effect with a model employing a purely isotropic long-range potential in combination with an angle dependent barrier that is zero between $\gamma=0^{\circ}$ and the cutoff angle $\gamma=\gamma_{c}$. 


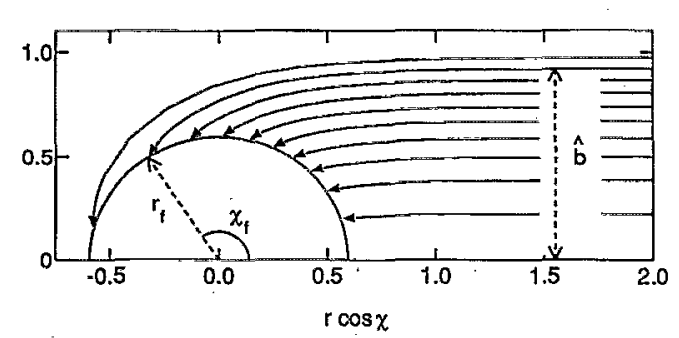

FIG. 1. High impact parameter (b) trajectories can be trapped by an isotropic long-range potential at low translational energy, thus washing out possible steric effects. In this figure $\hat{b}, r$, and $r_{f}$ are reduced quantities, i.e., $\hat{b}=b / b_{\max }(E), r=R / b_{\max }$, and $r_{f}=R_{f} / b_{\max }$, where $b_{\max }$ is the largest impact parameter that can lead to reaction. The subscript $f$ means at the harpooning radius and $R$ and $\chi$ are the space-fixed polar coordinates of the line of centers.

This gives an energy independent cone of reaction. It might seem surprising that this model can result in an energy dependent steric effect. The key to understanding this is that the orientation specified by $\gamma$ (as used in the ADLCM) is different from the orientation $\vartheta$ controlled by the experiment. Even if the interaction potential is zero, a purely geometric effect will make the angle of attack $\gamma$ different from the space fixed orientation $\vartheta$ for all trajectories with nonzero impact parameter. The presence of an attractive long-range isotropic potential will enlarge these differences. Particularly at low translational energy, trajectories with impact parameters $(b)$ larger than the radius of the imaginary shell $\left(R_{f}\right)$ will bend towards the molecule and "fly around" it to hit it at the back $\left(\gamma>90^{\circ}\right)$, as shown in Fig. 1. We call this "trapping." This effect will wash out the steric effect at low energies, or even make it negative.

The assumption of a barrierless reaction is not unrealistic for the $\mathrm{Ca}\left({ }^{1} D_{2}\right)+\mathrm{CH}_{3} \mathrm{~F}$ reaction. Experimentally, it was found that the total cross section increases at lower energies. This behavior is characteristic of a barrierless reaction with an attractive long-range potential ${ }^{13}$ and can easily be understood from the trapping model. Also, this model is consistent with the "harpooning mechanism" that has been proposed for this type of reaction. In this mechanism the reaction is initiated by an electron jump at a certain harpooning radius $R_{f}$, which is thought to correspond to the crossing of a covalent and an ionic surface. This mechanism thus gives a physical interpretation of the imaginary shell of the ADLCM, but it differs from the ADLCM in that the barrier is zero for a certain range of $\gamma$.

The difference between the experimentally controllable angle $\vartheta$ and the angle of attack $\gamma$ has been pointed out in literature several times. ${ }^{14-16}$ However, most of the attention is usually focused on $\gamma$. For example, an orientational opacity function has been defined in terms of $\gamma{ }^{7}$ In case there is a barrier to reaction, the trajectories with a relatively large impact parameter tend to hit the imaginary shell with a small radial component of the kinetic energy and are less likely to be reactive. Hence, in that case, reactive trajectories will have relatively small impact parameters and the distinction between $\gamma$ and $\vartheta$ is less important. On the other hand, for barrierless reactions we argue that the distinction between $\gamma$ and $\vartheta$ is the key to understanding the experiment. Therefore, in the sections below, we will cast the theory of the steric effect in terms of the experimentally relevant angle $\vartheta$.

In Sec. II we define the orientation dependent cross section in terms of $\vartheta$. This definition was first introduced by Stolte et al. ${ }^{17}$ in 1982 and has been used to report the experimental results. Following Stolte et al. we expand the orientation dependent cross section in Legendre moments $\left(\sigma_{i}\right)$, which have an appealing physical interpretation: $\sigma_{0}$ is the total cross section, $\sigma_{1} / \sigma_{0}$ is the orientation or steric effect and $\sigma_{2} / \sigma_{0}$ is the alignment effect.

In Sec. III we work out the theory for the computation of the orientation dependent cross section for isotropic potentials of the form

$$
V(R)=c R^{-n},
$$

with $n=1$...6. We include both the attractive $(c<0)$ and the repulsive $(c>0)$ case, where the former corresponds to a barrierless reaction and the latter to a reaction with a barrier. We will show the energy dependence of the first three Legendre moments $\left(\sigma_{0}, \sigma_{1} / \sigma_{0}\right.$, and $\left.\sigma_{2} / \sigma_{0}\right)$ for several values of the cutoff angle $\gamma_{c}$. This is possible because the result turns out not to depend on $R_{f}, c$, and $E$ separately, but rather on one dimensionless parameter, the reduced energy $\alpha=E R_{f}^{n} / c$. We only give results for $n=4$ since this corresponds to the leading term in the long range potential for $\mathrm{Ca}\left({ }^{1} D_{2}\right)+{ }_{n} \mathrm{CH}_{3} \mathrm{~F}$, The $R^{-4}$ dependence of the leading term in the $\mathrm{Ca}\left({ }^{1} D_{2}\right)+\mathrm{CH}_{3} \mathrm{~F}$ long range potential arises from the electrostatic interaction between the dipole moment of $\mathrm{CH}_{3} \mathrm{~F}$ and the quadrupole moments of the ${ }^{1} D$ substrates of $\mathrm{Ca}\left({ }^{1} D_{2}\right)$. For more details see Ref. 12.

In Sec. IV we discuss the case of a general anisotropic potential. In this case the rotation of the symmetric top molecule must be explicitly included in the model. One way to compute the orientation dependent cross section is by a standard quasiclassical trajectory (QCT) simulation of the experiment. We will show, however, that with this method the equations to obtain the Legendre moments $\sigma_{i} / \sigma_{0}$ for $i>1$. must be adapted in order to be consistent with the isotropic model. We present an alternative method which we call the modified quasiclassical trajectory (MQCT) method. This method yields again the same results for isotropic potentials, but we will argue that it is better for arbitrary anisotropic potentials. Furthermore, the MQCT method has numerical advantages.

We do not give numerical results for the anisotropic case. Rather, we will show the application of this theory to $\mathrm{Ca}\left({ }^{\mathrm{I}} \mathrm{D}_{2}\right)+\mathrm{CH}_{3} \mathrm{~F}$ in a separate paper. ${ }^{12}$ The reason for this is that the application to this system involves several issues, such as the choice of the potential and its asymptotic fivefold degeneracy, which are beyond the scope of the present paper.

\section{THE ORIENTATION DEPENDENT CROSS SECTION}

Information about the energy dependence of the steric effect is obtained by measuring the energy dependent, initial state selected, reactive cross sections $\sigma^{J K M}(E)$. The symmetric top quantum numbers are $J$, the total angular momentum, $M$, the projection of $\mathbf{J}$ onto the space fixed $\mathbf{z}$ axis (which is 
defined parallel to the homogeneous electric field) and $K$, the projection of $\mathbf{J}$ on the molecular symmetry axis $\left(\mathbf{z}^{\prime}\right)$. Different $M$ states (for given $J$ and $K$ ) have different average orientations

$$
\langle\cos \vartheta\rangle_{J K M}=\frac{K M}{J(J+1)}
$$

where $\vartheta$ is the angle between $\mathbf{z}$ and $\mathbf{z}^{\prime}$. The experimental setup is such that the relative velocity of the reagents is (approximately) parallel to the homogeneous electric field. Thus measuring $\sigma^{J K M}(E)$ for a set of $M$ values gives information about the orientation dependence of the reactive cross section. Since a given $M$ state does not correspond to a sharp value of $\rho \equiv \cos \vartheta$, but to a distribution of $\rho$ values $\mathscr{P}^{J K M}(\rho)$ (see Appendix A), we define the orientation dependent cross section $\sigma^{J K}(\dot{\rho}, E)$ implicitly by

$$
\sigma^{J K M}(E)=\int_{-1}^{1} \sigma^{J K}(\rho, E) \mathscr{P}^{J K M}(\rho) d \rho .
$$

Usually, the orientation dependent cross section is defined in the context of a classical model in which the rotation of the molecule is decoupled from the motion of the approaching atom. ${ }^{18}$ In such a model, $\sigma^{J K}(\rho, E)$ arises as the reactive cross section for a nonrotating molecule with a fixed orientation $\rho=\cos \vartheta$. In that case, the initial distribution $\mathscr{P}^{J K M}(\rho)$ remains unchanged during the approach of atom and Eq. (3) can be used to compute $\sigma^{J K M}(E)$ as a weighted average of $\sigma^{J K}(\rho, E)$. In our definition $\sigma^{J K}(\rho, E)$ does not arise from any specific model, but is defined as a function that satisfies Eq. (3) for known $\sigma^{J K M}(E)$ and $\mathscr{g}^{J K M}(\rho)$. For given values of $J$ and $K$ there are only $2 J+1$ possible $M$ values $(M=-J$, $-J+1, \ldots, J)$ and there could still be an infinite number of functions $\sigma^{J K}(\rho, E)$ satisfying Eq. (3). We fix $\sigma^{J K}(\rho, E)$ by the additional requirement that it is a linear combination of $2 J+1$ Legendre polynomials,

$$
\sigma^{J K}(\rho, E)=\sum_{l=0}^{2 J} \sigma_{l}^{J K}(E) P_{l}(\rho) .
$$

The probability distribution function $\mathscr{P}^{J K M}(\rho)$ can also be expanded in Legendre polynomials

$$
\bigoplus^{J K M}(\rho)=\sum_{l=0}^{2 J} c_{l}^{J K M} P_{l}(\rho) .
$$

The expansion coefficients $c_{l}^{J K M}$ are known analytically (see Appendix A). By substituting Eqs. (4) and (5) into Eq. (3) and integrating over $\rho$, we obtain the following set of $2 J+1$ linear equations relating the Legendre moments $\left\{\sigma_{l}^{\prime K}(E) ; l=0,1, \ldots, 2 J\right\}$ to the reactive cross sections $\left\{\sigma^{J K M}(E) ; M=-J,-J+1, \ldots, J\right\}:$

$$
\sigma^{J K M}(E)=\sum_{l=0}^{2 J} \sigma_{l}^{J K}(E) c_{l}^{J K M} \frac{2}{2 l+1} .
$$

Inversion of these linear equations for $J=K=1$ leads to the well known ${ }^{6}$ expressions for the Legendre moments in terms of the reactive cross sections

$$
\begin{aligned}
& \sigma_{0}^{1,1}(E)=\sigma^{1,1}(E), \\
& \frac{\sigma_{1}^{1,1}(E)}{\sigma_{0}^{1,1}(E)}=\frac{\sigma^{1,1,1}(E)-\sigma^{1,1,-1}(E)}{\sigma^{1,1}(E)}, \\
& \frac{\sigma_{2}^{1,1}(E)}{\sigma_{0}^{1,1}(E)}=5\left[\frac{\sigma^{1,1,1}(E)+\sigma^{1,1,-1}(E)}{\sigma^{1,1}(E)}-2\right] .
\end{aligned}
$$

Here, $\sigma^{J K}(E)=\sigma_{0}^{J K}(E)$ denotes the cross section for the unoriented molecules, which is equal to

$$
\sigma^{J K}(E)=\frac{1}{2 J+1} \sum_{M=-J}^{J} \sigma^{J K M}(E) .
$$

Thus the zeroth Legendre moment is equal to $\sigma^{1,1}(E)$ and we use it to normalize the other Legendre moments. It is advantageous to use $\sigma^{J K}(E)$ rather than $\sigma^{J K M}(E)$ with $M=0$, because the former is more easily accessible experimentally.

In Sec. III we consider an isotropic interaction potential, which decouples the rotation of the molecule from the motion of the atom. As a result, the ADLC type model described in Sec. I leads to an expression for the cross sections $\sigma^{J K M}(E)$ which has the form of Eq. (3) and we obtain an expression for $\sigma^{J K}(\rho, E)$ in a straightforward manner. The results from this Section could also have been obtained if $\sigma^{J K}(\rho, E)$ had been defined as the reactive cross section for a nonrotating molecule.

By contrast, in Sec. IV we consider an anisotropic interaction potential that can reorient the molecule. Computing $\sigma^{J K}(\rho, E)$ from trajectories that have initially nonrotating molecules would not give meaningful results, since the response of a nonrotating molecule to a torque is different from the response of a rotating molecule. One way to proceed would be to replace the reactive cross sections $\sigma^{J K M}(E)$ in Eqs. (7)-(9) by their standard quasiclassical approximations $\bar{\sigma}^{j k m}(E)$ (where $j, k$, and $m$ are the classical analogs of $J, K$, and $M$-see Sec. IV A). However, in the model for reaction we made the assumption that the angle of attack $\gamma$ and therefore indirectly the orientation of the molecule $\vartheta$-determines whether reaction occurs or not. Thus, we think that instead of using $m$ (the moment conjugate to $\phi$ ) to make the connection between classical and quantum mechanics, we should use the orientation dependent cross section $\sigma^{J K}(\rho, E)$ to make this connection. In other words: if we would make the correspondence $\sigma^{J K M}(E) \approx \bar{\sigma}^{j k m}(E)$ we would ignore the fact that the classical distribution of orientations $\overline{\mathscr{D}}^{j k m}(\rho)$ differs considerably from the quantum mechanical distribution $\mathscr{S}^{J K M}(E)$. Therefore, we propose to compute a quasiclassical approximation $\bar{\sigma}^{j k}(\rho, E)$ to the orientation dependent cross section by solving the classical ana$\log$ of Eq. (3)

$$
\bar{\sigma}^{j k m}(E)=\int_{-1}^{1} \bar{\sigma}^{j k}(\rho, E) \overline{\mathscr{P}}^{j k m}(\rho) d \rho .
$$

In Sec. IV we present two methods to solve $\bar{\sigma}^{j k}(\rho, E)$ from this equation. In the first method we only use trajectories that start with $m$ values that correspond to the quantum mechanical values $M$. Since we do not make the connection $\sigma^{J K M}(E) \approx \bar{\sigma}^{j k m}(E)$ but instead $\sigma^{J K}(\rho, E) \approx \bar{\sigma}^{j k}(\rho, E)$, we 


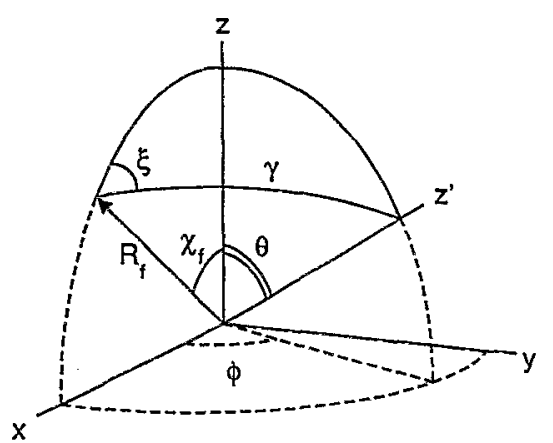

FIG. 2. The atom hits the imaginary sphere at the point determined by the polar angle $\chi$ and the azimuthal angle $\phi$. The polar angle of the symmetry axis of the molecule is $\vartheta$. The reaction probability is a function of the angle of attack $\gamma$. In Eq. (19) the integration over $\vartheta$ and $\phi$ is replaced by the integration over $\gamma$ and $\xi$.

have complete freedom in the choice of $m$ and in the second method, which we will refer to as the modified quasiclassical trajectory (MQCT) method, we use all classically allowed $m$ values. The MQCT method can be viewed as an alternative method to quantize $m$ : we compute $\bar{\sigma}^{j k}(\rho, E)$ from Eq. (11) using all possible $m$ values, and substitute it back into Eq. (3) to obtain approximations to $\sigma^{J K M}(E)$ for quantum mechanical $M$ values.

\section{THE ISOTROPIC CASE}

In the case of an isotropic interaction potential between the symmetric top molecule and the atom, the motion of the centers of mass of the molecule and the atom decouple from the rotation of the molecule. Therefore, we can describe this rotation quantum mechanically, using the probability function $\oiint^{J K M}(\phi, \vartheta, \psi)$ defined in Eq. (A3) and treat the centers of mass motion classically. We choose a space-fixed coordirate system with its origin in the center of mass of the atommolecule system. The $z$ axis is chosen parallel to the initial relative velocity. The vector connecting the centers of mass is denoted by $\mathbf{R}$. We define the space-fixed $x$ axis by requiring $\mathbf{R}$ to lie in the $(x, z)$ plane initially. The impact parameter is $b$, thus initially $\mathbf{R}_{i}=(b, 0, \infty)$. The centers of mass motion will be described using polar coordinates $R=|\mathbf{R}|$ and $\chi$ (the angle between $\mathbf{R}$ and the space-fixed $\mathbf{z}$ axis). The orientation of the molecule is described using the Euler angles $(\phi, \vartheta, \psi)$ in the space-fixed frame. The molecular symmetry axis is

$$
\mathbf{z}^{\prime}=\left[\begin{array}{c}
\sin \vartheta \cos \phi \\
\sin \vartheta \sin \phi \\
\cos \vartheta
\end{array}\right]
$$

We propagate $(R, \chi)$ until $R$ reaches some fixed final value $R_{f}$. The final angle $\chi_{f}$ depends on the impact parameter and on the translational energy $E: \chi_{f}=\chi_{f}(b, E)$. Reaction is assumed to occur if the angle of attack $(\gamma)$ between $\mathbf{R}_{f}$ and the molecular symmetry axis $\mathbf{z}^{\prime}$ is less than a critical value $\gamma_{c}$ (the angles are shown in Fig. 2). Introducing the reaction probability

$$
W(\gamma)= \begin{cases}1 ; & 0 \leqslant \gamma \leqslant \gamma_{c} \\ 0 ; & \gamma_{c}<\gamma \leqslant \pi\end{cases}
$$

we can write the reactive cross section as

$$
\begin{aligned}
\sigma^{J K M}(E)= & 2 \pi \int_{0}^{b_{\max }(E)} b d b \int_{0}^{2 \pi} d \phi \int_{0}^{2 \pi} d \psi \int_{-1}^{1} d \cos \vartheta \\
& \times \mathcal{P}^{J K M}(\phi, \vartheta, \psi) W\left\{\gamma\left[\chi_{f}(b, E), \phi, \vartheta\right]\right\},
\end{aligned}
$$

where $b_{\max }(E)$ must be equal to or larger than the largest impact parameter that can lead to reaction. Using Eqs. (A3)(A5) we can perform the integration over $\psi$, which gives

$$
\begin{aligned}
\sigma^{J K M}(E)= & \int_{0}^{b_{\max }(E)} b d b \int_{0}^{2 \pi} d \phi \int_{-1}^{1} d \cos \vartheta \\
& \times \operatorname{Pos}^{J K M}(\cos \vartheta) W\left\{\gamma\left[\chi_{f}(b, E), \phi, \vartheta\right]\right\} .
\end{aligned}
$$

Comparing this equation to Eq. (3) we find for the orientation dependent reactive cross section

$$
\sigma^{J}(\rho, E)=\int_{0}^{b_{\max }(E)} b d b \int_{0}^{2 \pi} d \phi W\left\{\gamma\left[\chi_{f}(b, E), \phi, \vartheta\right]\right\}
$$

and for its Legendre moments [using Eqs. (A7) and (4)]

$$
\begin{aligned}
\sigma_{l}^{J K}(E)= & \frac{2 l+1}{2} \int_{0}^{b_{\max }(E)} b d b \int_{0}^{2 \pi} d \phi \int_{-1}^{1} d \cos \vartheta \\
& \times P_{l}(\cos \vartheta) W\left\{\gamma\left[\chi_{f}(b, E), \phi, \vartheta\right]\right\} .
\end{aligned}
$$

The discontinuity in $W(\gamma)$ makes it difficult to evaluate the integral. This problem can be removed by the following change of variables $(\vartheta, \phi) \rightarrow(\gamma, \xi)$. Here $\xi$ is the angle between the $(x, z)$ plane and the plane through $\mathbf{R}_{f}$ and $\mathbf{z}^{\prime}$ [see Fig. (2)]. Thus $\gamma$ and $\xi$ are the polar angles of the $\mathbf{z}^{\prime}$ axis in a frame which arises from rotating the space fixed frame around the $y$ axis over an angle $\chi_{f}$. Hence, we can replace

$$
\int_{-1}^{1} d \cos \vartheta \int_{0}^{2 \pi} d \phi=\int_{-1}^{1} d \cos \gamma \int_{0}^{2 \pi} d \xi
$$

We now eliminate $W$ from Eq. (18) by limiting the range of integration for $\gamma$

$$
\begin{aligned}
\sigma_{l}^{J K}(E)= & \frac{2 l+1}{2} \int_{0}^{b_{\max }(E)} b d b \int_{0}^{2 \pi} d \xi \int_{\cos \gamma_{c}}^{1} d \cos \gamma \\
& \times P_{l}\left\{\cos \vartheta\left[\chi_{f}(b, E), \gamma, \xi\right]\right\} .
\end{aligned}
$$

The expression for $\cos \vartheta$ is

$$
\cos \vartheta=\sin \chi_{f} \cos \xi+\cos \chi_{f} \cos \gamma .
$$

All that remains to be done before we can evaluate this integral is to derive formulas for $\chi_{f}(b, E)$ and $b_{\max }(E)$. Of course, these functions depend on the shape of the potential. However, we will first draw a few conclusions that are independent of $V(R)$.

\section{A. Special cases}

First, we note that in the current model the Legendre moments [Eq. (20)] are independent of $J$ and $K$ and in the 
remainder of this section, we will drop those labels [N.B. the reactive cross sections $\sigma^{J K M}(E)$ still depend on $J$ and $K$ because the probability density functions $\operatorname{PJ}^{J K M}(\rho)$ are $J, K$ dependent, see Eq. (3)].

For $l=0$ we can evaluate the integral analytically and we obtain the following simple expression for the total reactive cross section:

$$
\sigma_{0}(E)=\pi b_{\max }^{2}(E)\left(\frac{1-\cos \gamma_{c}}{2}\right) .
$$

In the limit of large energy $[E \gg V(R)]$ we have $b_{\max }=R_{f}$ and find the completely intuitive result that the reactive cross section is equal to a collisional cross section multiplied by a factor between zero and one that depends on the size of the cone of reaction.

For $l=1$ we derive (without approximations)

$\frac{\sigma_{1}(E)}{\sigma_{0}(E)}=3\left(1+\cos \gamma_{c}\right) \frac{1}{b_{\max }^{2}} \int_{0}^{b_{\max }(E)} \cos \chi_{f}(b, E) b d b$.

Again we can take the limit for large $E$, in which case cos $\chi_{f}$ can be determined by the geometric relation

$$
\cos \chi_{f}(b)=\sqrt{1-\left(b / b_{\max }\right)^{2}}
$$

and we obtain

$$
\lim _{E \rightarrow \infty} \frac{\sigma_{1}(E)}{\sigma_{0}(E)}=1+\cos \gamma_{c} .
$$

Hence, in the limit of high energy the steric effect must be positive and have a maximum of two. Note that the high energy limit actually applies to any potential, even to anisotropic ones.

Before we proceed to derive the general formulas we will give a lower and upper bound for the steric effect valid for arbitrary energies. These values are obtained by setting $\chi_{f}$ to $\pi$ and 0 in Eq. (23), respectively. The upper limit can actually be approached in the case of a repulsive potential at low energies, in which case only small impact parameter trajectories are reactive. One expects always to stay clear of the lower limit

$$
-\frac{3}{2}\left(1+\cos \gamma_{c}\right)<\frac{\sigma_{1}(E)}{\sigma_{0}(E)} \leqslant \frac{3}{2}\left(1+\cos \gamma_{c}\right) .
$$

Note that these limits rely on the assumption of an isotropic potential.

\section{B. General solution}

The general expressions for $\chi_{f}(b, E)$ and $b_{\max }(E)$ are found by solving the classical equations of motion of the centers of mass of the atom-molecule system. The solution of this effective central force problem is well known. ${ }^{13,19}$ For the deflection angle at $R_{f}$ we find

$$
\chi_{f}(b, E)=\int_{R_{f}}^{\infty} \frac{b}{\sqrt{R^{4}-b^{2} R^{2}-R^{4} V(R) E^{-1}}} d R .
$$

We derive the expression for $b_{\max }(E)$ in the usual way by considering the effective potential ${ }^{13}$ [see Eq. (1)]

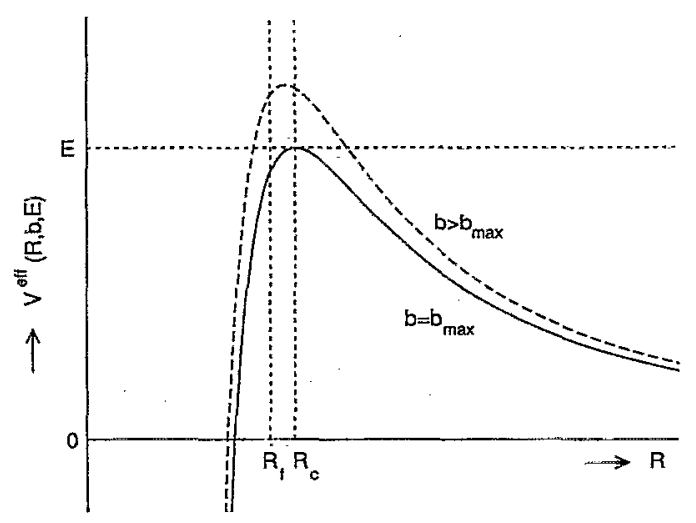

FIG. 3. The effective potential.

$$
V^{\mathrm{eff}}(R, b, E)=\frac{b^{2} E}{R^{2}}+\frac{c}{R^{n}} .
$$

We find $b_{\max }$ from condition (I)

$$
V^{\text {eff }}\left(R_{f}, b_{\max }, E\right)=E \text {. }
$$

However, we must be aware that for $c<0$ and $n \geqslant 3$ the effective potential has a maximum at

$$
R_{c}(b, E)=\left(\frac{-n c}{2 b^{2} E}\right)^{1 /(n-2)}
$$

as shown in Fig. (3). Hence, if $R_{f}<R_{c}(b, E)$, then $b_{\max }$ is found from condition (II)

$$
V^{\text {eff }}\left[R_{c}\left(b_{\max }, E\right), b_{\max }, E\right]=E \text { :- }
$$

Condition (I) gives

$$
b_{\max }^{(\mathrm{I})}=R_{f} \sqrt{1-\frac{c}{E R_{f}^{n}}}
$$

and condition (II) leads to

$$
b_{\max }^{(\mathrm{II})}=\left(\frac{-c}{E}\right)^{1 / n}\left(\frac{n}{2}\right)^{1 / 2}\left(\frac{n}{2}-1\right)^{(2-n) /(2 n)},
$$

and also

$$
R_{c}(E, n, c)=\left(\frac{-c}{E}\right)^{1 / n}\left(\frac{n}{2}-1\right)^{1 / n}
$$

Note that this last result is identical to the Langevin model. ${ }^{13}$ Thus we are in regime (II) if all of the following three conditions are satisfied:
(1) $c<0$
(2) $n \geqslant 3$;
(3) $R_{f}<R_{c}(E, n, c)$,

and otherwise we are in regime (I).

We now have presented all the formulas needed to compute the Legendre moments $\sigma_{l}$ of Eq. (20) for a given $E, R_{f}$, $c, n$, and $\gamma_{c}$. It turns out, however, that by introducing the reduced energy

$$
\alpha=E \frac{R_{f}^{n}}{c} \text {. }
$$

the Legendre moments can be written as 


$$
\sigma_{l}\left(E, n, c, R_{f}, \gamma_{c}\right)=\pi b_{\max }^{2}\left(\alpha, n, R_{f}\right) s_{l}\left(\alpha, n, \gamma_{c}\right),
$$

and as a result, $\sigma_{l} / \sigma_{0}$ depends on three parameters only $\left(\alpha, n, \gamma_{c}\right)$. This is an extremely important result, since it allows us to easily examine the behavior of our model for the entire parameter space. For $\sigma_{0}(E)$ we actually have a simple closed formula (see below). which depends on three parameters $\left(\alpha, R_{f}, \gamma_{c}\right)$ in regime (I) and on four parameters $\left(\alpha, R_{f}, \gamma_{c}, n\right)$ in regime (II).

First, using the equations for $R_{c}$ and $\alpha$ [Eqs. (34) and (35)] we rewrite the three conditions that determine when we are in regime (II) in the compact form

$$
1-\frac{n}{2}<\alpha<0
$$

For $b_{\max }$ we find the expressions

$$
\begin{aligned}
& b_{\max }^{(\mathrm{l})}\left(\alpha, R_{f}\right)=R_{f}\left(1-\frac{1}{\alpha}\right)^{1 / 2} \\
& b_{\max }^{(\mathrm{I})}\left(\alpha, R_{f}, n\right)=R_{f}(-\alpha)^{-1 / n}\left(\frac{n}{2}\right)^{1 / 2}\left(\frac{n}{2}-1\right)^{(2-n) /(2 n)}
\end{aligned}
$$

Introducing the reduced impact parameter

$$
\tilde{b}=b / b_{\max },
$$

we find for the last factor in Eq. (36), using Eq. (20)

$$
\begin{aligned}
s_{l}\left(\alpha, n, \gamma_{c}\right)= & \frac{2 l+1}{2} \int_{0}^{1} \tilde{b} d \tilde{b} \int_{0}^{2 \pi} d \xi \int_{\cos \gamma_{G}}^{1} d \cos \gamma \\
& \times P_{l}\{\cos \vartheta[\chi(\tilde{b}, E), \xi, \gamma]\},
\end{aligned}
$$

where $\chi(\vec{b}, E)$ is given by

$$
\chi_{f}(\tilde{b}, E)=\int_{r_{f}}^{\infty} \frac{\tilde{b}}{\sqrt{r^{4}-b^{2} r^{2}-r^{4-n} \alpha^{-1} r_{f}^{n}}} d r .
$$

Here, $r_{f}$ is the reduced final radius

$$
r_{f}=R_{f} / b_{\max }
$$

which is expressed as a function of $\alpha$ (in regime I) or $\alpha$ and $n$ (in regime II) using Eqs. (38) and (39), respectively. For numerical evaluation of this integral it is convenient to map the infinite range $\left[r_{f}, \infty\right]$ onto $\left[0,1 / r_{1}\right]$ by the substitution $y=1 / r$. This gives our final formula for $\chi_{f}$

$$
\chi_{f}(\tilde{b}, E)=\int_{0}^{r_{f}^{-1}} \frac{\tilde{b}}{\sqrt{1-\tilde{b}^{2} y^{2}-y^{n} \alpha^{-1} r_{f}^{n}}} d y .
$$

Summarizing, Eqs. (21) and (35)-(44) are all we need to compute $\sigma_{l}(E) / \sigma_{0}(E)$. We wrote a small Fortran program to compute the integrals of Eqs. (41) and (44), using the NAG library $^{20}$ routines D01AJF for the integral over $\tilde{b}$, D01DAF for the integrals over $\xi$ and $\cos (\gamma)$ and D01ATF for the integral over $y$. In addition, we made one more substitution to facilitate the numerical evaluation of the integral; in Eq. (41) we substitute $g=\tilde{b}^{2}$ giving

$$
\int_{0}^{1} \tilde{b} d \tilde{b}=\frac{1}{2} \int_{0}^{1} d g .
$$
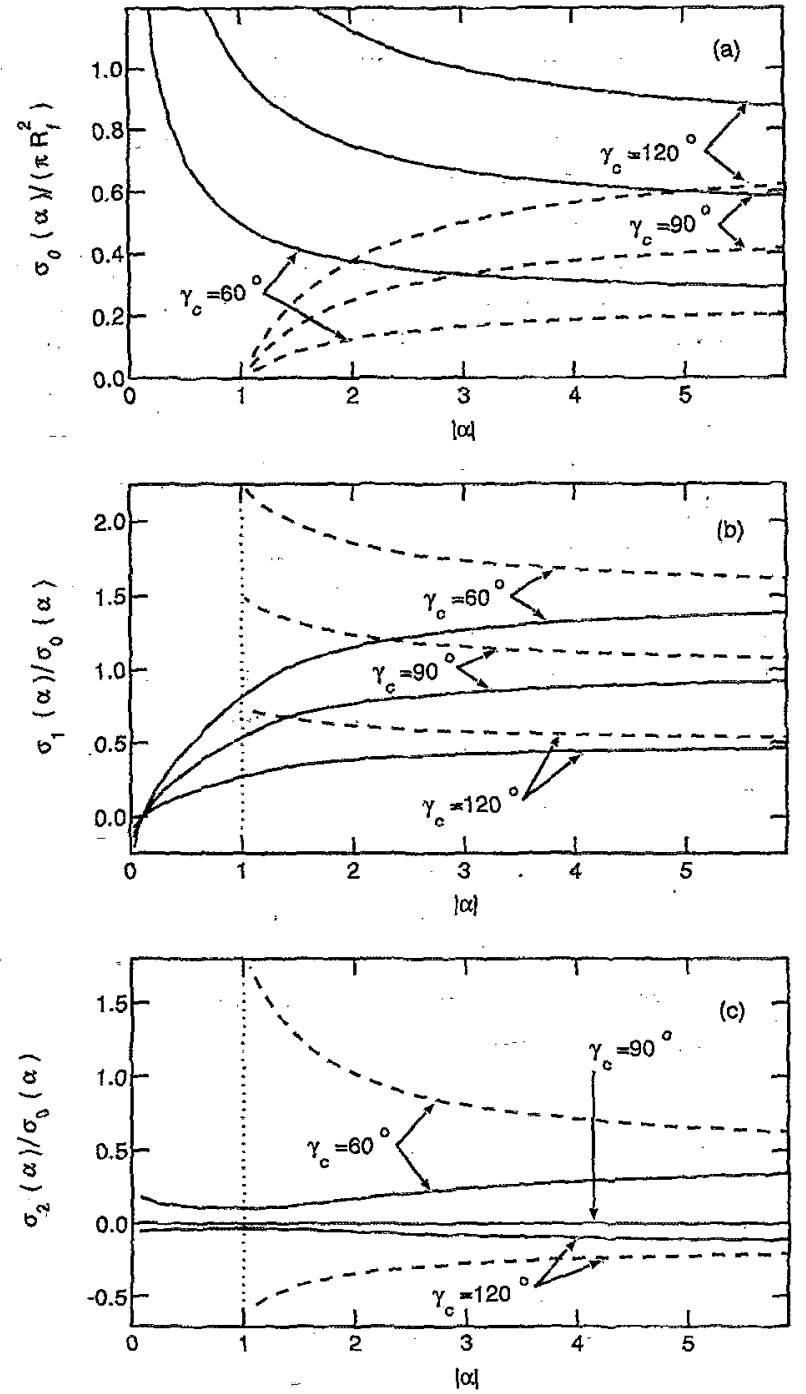

FIG. 4. The reactive cross section for unoriented molecules [panel (a)], the steric effect [panel (b)], and the alignment effect [panel (c)], as a function of the reduced energy $\alpha$ for several values of the critical angle of attack $\gamma_{c}$.

Because of all the transformations the integrands are well behaved and the required computer time is negligible.

To compute $\sigma_{0}(E)$ no integrals have to be evaluated, Eqs. (22), (38), and (39) suffice.

\section{Results}

In Fig. 4 we show the energy dependence of the total reactive cross section, the steric effect and the alignment effect for a $c R^{-n}, n=4$, potential. The solid curves correspond to an attractive potential $[\alpha<0, c<0$, see Eq. (35)] and the dashed curves to a repulsive potential $(\alpha>0)$. We give results for cutoff angles $\gamma_{c}$ of 60,90 , and 120 degrees.

Panel (a) gives the total reactive cross section normalized to the high energy collisional cross section $\left(\pi R_{f}^{2}\right)$. At high energy we have $b_{\max }=R_{f}$, thus from Eq. (22) we know that the curves, independently of the sign of $\alpha$, should converge to $1 / 4,1 / 2$, and $3 / 4$ for $\gamma_{c}=60,90$, and 120 degrees, 
respectively. For repulsive potentials, the reactive cross section is zero for $\alpha<1$, since the total energy is less than the potential at the harpooning radius $R_{f}$ in that case [see Eq. (35)]. We see that in all cases an attractive potential results in a negative energy dependence of the total reactive cross section and a repulsive potential in a positive energy dependence.

In panel (b) we show the steric effect $\sigma_{1} / \sigma_{0}$. The most important conclusion is that the energy dependence is exactly opposite to the energy dependence of $\sigma_{0}$ : It is positive for attractive potentials and negative for repulsive potentials. Furthermore, the larger the cutoff angle $\left(\gamma_{c}\right)$, the smaller the steric effect. In agreement with Eq. (25) the high energy limits are $3 / 2,1$, and $1 / 2$ for $\gamma_{c}=60^{\circ}, 90^{\circ}$, and $120^{\circ}$, respectively. We reach the upper limit given in Eq. (26) for repulsive potentials near $\alpha=1$. The lower limit given in the same equation is not reached, but at low energies $(|\alpha|<0.11$, approximately) we actually get a negative steric effect: because of the trapping, as shown in Fig. 1, trajectories are more likely to hit tails than heads.

Finally, panel (c) shows the alignment effect $\sigma_{2} / \sigma_{0}$. For attractive potentials it is small and nearly energy independent. For $\gamma_{c}=90^{\circ}$ it is identically zero at all energies for both the repulsive and the attractive potentials. For repulsive potentials the alignment effect is much more sensitive to the cutoff angle than for attractive potentials, but in any case it is positive for $\gamma_{c}>90^{\circ}$ and negative for $\gamma_{c}<90^{\circ}$.

\section{THE ANISOTROPIC CASE}

We present two methods to obtain the quasiclassical orientation dependent cross section $\bar{\sigma}^{j k}(\rho, E)$ from Eq. (11). Both methods have the desirable property that they yield the same result as the method described in Sec. II in the case of an isotropic potential. For a general potential they might give different results, and below we will argue why we prefer the second method. Before we describe the two methods we must give a brief introduction into the classical description of a symmetric top.

\section{A. Classical description of a symmetric top}

The orientation of the symmetric top is given by the three Euler angles $(\phi, \vartheta, \psi)$. The moments conjugate to these angles are $p_{\phi}, p_{\vartheta}$, and $p_{\psi}$. The symmetric top classical Hamiltonian is given by

$$
H_{\text {rot }}=A \frac{\left(p_{\phi}-p_{\psi} \rho\right)^{2}}{1-\rho^{2}}+A p_{\vartheta}^{2}+C p_{\psi}^{2}
$$

where $A$ and $C$ are the rotational constants. From Hamilton's classical equations of motion we have

$$
\begin{aligned}
& \dot{p}_{\phi}=-\frac{\partial H}{\partial \phi}=0, \\
& \dot{p}_{\psi}=-\frac{\partial H}{\partial \psi}=0,
\end{aligned}
$$

and thus $p_{\phi}$ and $p_{\psi}$ are constants of the motion and we define $p_{\phi}=m$ and $p_{\psi}=k$. For a total angular momentum $j$ the energy is

$$
E_{\mathrm{rot}}=A j^{2}+(C-A) k^{2} \text {. }
$$

Solving $H=E$ for $p_{\vartheta}$ gives

$$
p_{\vartheta}^{2}=j^{2}-k^{2}-\frac{(m-k \rho)^{2}}{1-\rho^{2}}
$$

and we derive the classical distribution

$$
\bar{\rho}^{j k m}(\rho) \sim \frac{1}{|\dot{\rho}|} \sim \frac{1}{\left|p_{\vartheta} \sin \vartheta\right|} .
$$

With the appropriate normalization we have

$$
\operatorname{jos}^{j k m}(\rho)=\left\{\begin{array}{l}
\frac{1}{\pi}\left(-\rho^{2}+2 \frac{m k}{j^{2}} \rho+1-\frac{m^{2}}{j^{2}}-\frac{k^{2}}{j^{2}}\right)^{-1 / 2}, \\
\text { for } \rho_{1}<\rho<\rho_{2}, \\
0 \quad \text { otherwise, }
\end{array}\right.
$$

where

$$
\rho_{1,2}=\frac{m k}{j^{2}} \mp \sqrt{\left(1-\frac{k^{2}}{j^{2}}\right)\left(1-\frac{m^{2}}{j^{2}}\right)} .
$$

This distribution function has been obtained by Choi et al. ${ }^{21}$ from geometrical arguments. The quasiclassical approximation of the state $|J K M\rangle$ is obtained by setting

$$
\begin{aligned}
& j=\hbar \sqrt{J(J+1)}, \\
& m=\hbar M, \\
& k=\hbar K .
\end{aligned}
$$

If we follow the derivation in Sec. III using Eq. (11) to define $\bar{\sigma}^{j k}(\rho, E)$ we obtain an expression for $\bar{\sigma}^{j k}(\rho, E)$ identical to Eq. (17). In other words, we have $\bar{\sigma}^{j k}(\rho, E)=\sigma^{J K}(\rho, E)$ for isotropic potentials. We may now define the two methods that apply to the anisotropic case.

\section{B. Quasiclassical trajectory (QCT) method}

The quasiclassical reactive cross sections $\bar{\sigma}^{j k m}(E)$ are obtained by trajectory calculations. We have the following initial conditions: $\phi$ and $\psi$ are sampled uniformly, $\cos \vartheta$ is sampled according to distribution Eq. (52) and for the impact parameter we have $P(b) \sim b, 0<b<b_{\max }$. The conjugate momenta are given by Eqs. (50), (55), and (56). For each trajectory the classical equations of motion are integrated numerically and we determine the final angle $\gamma$. The reaction probability $W(\gamma)$ as defined in Eq. (13) determines whether a trajectory is reactive or not. The reactive cross section is approximated by

$$
\bar{\sigma}^{j k m}(E)=\frac{N_{r}(E)}{N_{t}} \pi b_{\max }^{2}
$$

where $N_{r}(E)$ is the number of reactive trajectories and $N_{t}$ the total number of trajectories. $b_{\max }$ must be chosen large enough not to miss any reactive trajectories, $N$ must be increased to improve the accuracy.

We expand the classical distribution in Legendre polynomials 


$$
\tilde{D}^{j k m}(\rho)=\sum_{l=0}^{\infty} \bar{c}_{l}^{j k m} \mathscr{P}_{l}(\rho)
$$

For the expansion coefficients we have an analytic expression (see Appendix B)

$$
\bar{c}_{l}^{j k m}=\frac{2 l+1}{2} P_{l}\left(\frac{k}{j}\right) P_{l}\left(\frac{m}{j}\right)
$$

We can now compute the Legendre moments of the orientation dependent reactive cross section $\bar{\sigma}^{j k}(\rho, E)$ from the classical analog of Eq. (6).

Just as in Sec. II we can replace the equation with $m=0$ by the equation for the unoriented reactive cross section. For this purpose we must generate trajectories with random $m$ values $m \in[-j, j]$. The corresponding distribution in $\rho$ is indeed uniform

$$
\hat{p}^{j k}(\rho)=\frac{1}{2 j} \int_{-j}^{j} \bar{\rho}^{j k m}(\rho) d m=\frac{1}{2}
$$

For the example $J=K=1$ we now find

$$
\begin{aligned}
& \bar{\sigma}_{0}^{1,1}(E)=\bar{\sigma}^{1,1}(E), \\
& \frac{\bar{\sigma}_{1}^{1,1}(E)}{\bar{\sigma}_{0}^{1,1}(E)}=\frac{\bar{\sigma}^{1,1,1}(E)-\bar{\sigma}^{1,1,-1}(E)}{\bar{\sigma}^{1,1}(E)}, \\
& \frac{\bar{\sigma}_{2}^{1,1}(E)}{\bar{\sigma}_{0}^{1,1}(E)}=8\left[\frac{\bar{\sigma}^{1,1,1}(E)+\bar{\sigma}^{1,1,-1}(E)}{\bar{\sigma}^{1,1}(E)}-2\right] .
\end{aligned}
$$

Comparing this to Eqs. (7)-(9) we find that only the expression for the alignment effect is different from the quantum version.

\section{Modified quasiclassical trajectory (MQCT) method}

This method only requires the computation of trajectories with random $m$ [which are also required for the computation of $\sigma_{0}(E)$ in the QCT method]. We introduce the expansion

$$
\bar{\sigma}^{j k m}(E)=\sum_{l=0}^{\infty} \bar{c}_{l}^{j k}(E) P_{l}\left(\frac{m}{j}\right)
$$

which gives

$$
\bar{c}_{l}^{j k}(E)=\frac{\int_{-1}^{1} d(m / j) P_{l}(m / j) \tilde{\sigma}^{j k m}(E)}{\int_{-1}^{1} d(m / j) P_{l}^{2}(m / j)} .
$$

Using the Monte Carlo approximation to the integral

$$
\int_{a}^{b} f(x) d x=\frac{b-a}{N} \sum_{i=1}^{N} f\left(x_{i}\right),
$$

we find

$$
\bar{c}_{l}^{j k}(E)=\pi b_{\max }^{2} \frac{2 l+1}{2} \frac{2}{N_{t}} \sum_{i} P_{l}\left(\frac{m_{i}}{j}\right),
$$

where the summation is only over the reactive trajectories. If we substitute expansion (64) together with

$$
\bar{\sigma}^{j k}(\rho, E)=\sum_{l=0}^{\infty} \bar{\sigma}_{l}^{j k}(E) P_{l}(\rho)
$$

into Eq. (11), we find

$$
\sum_{l=0}^{\infty} \bar{c}_{l}^{j k}(E) P_{l}\left(\frac{m}{j}\right)=\int_{-1}^{1} \sum_{l^{\prime}=0}^{\infty} \bar{\sigma}_{l^{\prime}}^{j k}(E) P_{l}(\rho) \overline{\mathscr{P}}^{j k m}(\rho) d \rho .
$$

Multiplying this equation with $P_{l}(m / j)$ and integrating over $m / j$ gives

$$
\bar{c}_{l}^{j k}(E)=\sum_{l^{\prime}=0}^{\infty} A_{l l^{\prime}}(j, k) \bar{\sigma}_{l^{\prime}}^{j k}(E)
$$

where

$A_{l l^{\prime}}(j, k)=\frac{2 l^{\prime}+1}{2} \int_{-1}^{1} d \frac{m}{j} \int_{-1}^{1} d \rho P_{l}\left(\frac{m}{j}\right) \operatorname{Sojkm}^{j k}(\rho) P_{l^{\prime}}(\rho)$.

Surprising as it may seem, these matrix elements can be evaluated analytically (see Appendix B) and the result is

$$
A_{l l^{\prime}}(j, k)=P_{l}\left(\frac{k}{j}\right) \delta_{l l^{\prime}}
$$

Because of the Kronecker delta in this expression we can easily invert Eq. (70), giving the MQCT expressions for the Legendre moments of the orientation dependent reactive cross section

$$
\bar{\sigma}_{l}^{j k}(E)=\bar{c}_{l}^{j k}(E) / P_{l}\left(\frac{k}{j}\right) .
$$

Comparing the two methods we see that in the QCT method the steric effect is computed from the difference of the reactive cross section for two values of $m$ [Eq. (62)], whereas the MQCT method relies on all classically allowed $m$ values [Eqs. (67) and (73)]. The classical probability density function corresponding to a specific $m$ value is sharply peaked and very different from the smooth quantum mechanical probability density function (see, e.g., Fig. 1 in the accompanying paper ${ }^{12}$ or Fig. 4 in Ref. 21). Therefore, we expect the MQCT to sample the potential energy surface much more realistically than the QCT method. Numerically, the MQCT has the advantage that it only requires trajectories for random $m$ values, which are needed anyway to compute $\sigma_{0}(E)$.

Note that one cannot generate the quantum mechanical probability distribution by simply taking the initial $\rho$ values of the trajectories according to $\mathscr{P}^{J K M}(\rho)$. First of all, with $m$ fixed by the quantization condition Eq. (55), some trajectories would have to start in a classically forbidden region. Second, the resulting distribution of trajectories would not be stationary and the fraction of reactive trajectories would depend on $R(0)$. Lifting the quantization condition for $m$ makes it possible to generate stationary distributions of $\rho$ that differ from the classical distribution. Continuing along this line of thought led us to the development of the MQCT method.

Note, incidentally, that with the QCT method one computes at most $2 J+1$ Legendre moments, as in the quantum mechanical case, whereas with the MQCT method one can 
formally compute as many Legendre moments as desired. In practice, this has little relevance, since both methods are expected to be very sensitive to statistical errors for the higher Legendre moments.

\section{v. CONCLUSION}

It has been shown that a positive energy dependence of the steric effect does not require reorientation. We have presented a model with three elements, an imaginary shell (with radius $R_{f}$ ) surrounding the molecule at which reaction is assumed to occur, a critical angle $\gamma_{c}$ which determines which part of the shell is reactive, and an isotropic long range potential of the form $c R^{-n}$. This model predicts a positive energy dependence of the steric effect for attractive potentials and a negative one for repulsive potentials. For $\sigma_{0}(E)$ the energy dependence is reversed. For attractive potentials our model predicts a small, almost energy independent alignment effect. The computation of $\sigma_{l} / \sigma_{0}$ for isotropic potentials is numerically easy, since most of the work can be done analytically.

We also present two methods for the computation of $\sigma_{l} / \sigma_{0}$ for arbitrary (anisotropic) potentials. The first method is based on the standard quasiclassical trajectory (QCT) method for the computation of state selected reactive cross sections $\bar{o}^{j k m}(E)$. The most important conclusion with respect to this method is that the relation between $\sigma_{l} / \sigma_{0}$ for $l \geqslant 2$ and the reactive cross sections from QCT differ from the quantum mechanical version, because all but the zeroth and first Legendre moments of the classical distribution function $j^{j k m}(\rho)$ are different from the quantum mechanical ones.

The second method, which we refer to as the modified quasiclassical trajectory method (MQCT), is based on the computation of $\bar{\sigma}^{j k m}(E)$ for all classically allowed $m$ values, and not just from the discrete set used in the QCT. The coefficients of the expansion of $\bar{\sigma}^{j k m}(E)$ in Legendre polynomials in $(m / j)$ are directly related to the Legendre moments $\bar{\sigma}^{j k}(\rho, E)$ of the orientation dependent reactive cross section. We favor this MQCT method over the QCT method because we expect the MQCT method to sample the potential energy surface more evenly and thus give a better orientation dependent reactive cross section. For isotropic potentials both methods give the same result.

In the accompanying paper ${ }^{12}$ we will show that the parameters in the isotropic model needed to reproduce the experimental results for the energy dependence of the steric and alignment effects in the $\mathrm{Ca}\left({ }^{1} D_{2}\right)+\mathrm{CH}_{3} \mathrm{~F}$ reaction are physically reasonable. We will also study the effects of anisotropy in the potential using the MQCT method and we will compare the MQCT and QCT numerically.

\section{ACKNOWLEDGMENTS}

The authors thank Ad van der Avoird, Maurice H. M. Janssen, David H. Parker, Scott M. Auerbach, and Paul E. S. Wormer for giving very useful comments on the manuscript. We are also grateful to R. A. Kortram for helping us with the proof of Theorem 1 in Appendix B. This work was supported by the Royal Netherlands Academy of Arts and Sciences (KNAW). A.J.H.M.M. acknowledges financial support from the Netherlands Foundation for Chemical Research (SON) and the Netherlands Organization for the Advancement of Research (NWO).

\section{APPENDIX A}

The orientation of a symmetric top molecule with respect to the space-fixed frame is specified by the three Euler angles $(\phi, \vartheta, \psi)$. We use the zyz parametrization, the domain of the angles is $[0,2 \pi)$ for $\phi$ and $\psi$ and $[0, \pi]$ for $\vartheta$, the volume element is $d \tau=d \phi d \psi d \cos \vartheta$. Using active rotations, the wave function is given by ${ }^{22}$

$$
\begin{aligned}
\Psi^{J K M}(\phi, \vartheta, \psi) & =\left(\frac{2 J+1}{8 \pi^{2}}\right)^{1 / 2} D_{M K}^{J}(\phi, \vartheta, \psi)^{*} \\
& =\left(\frac{2 J+1}{8 \pi^{2}}\right)^{1 / 2} e^{i M \phi} d_{M K}^{J}(\vartheta) e^{i K \psi} .
\end{aligned}
$$

The probability function, i.e., the square modulus of the wave function

$$
\operatorname{sj}^{J M}(\phi, \vartheta, \psi)=\frac{2 J+1}{8 \pi^{2}}\left[d_{M K}^{J}(\vartheta)\right]^{2}
$$

thus only depends on $\vartheta$ and we define

$$
\begin{aligned}
\mathscr{D}^{J K M}(\rho) & \equiv \int_{0}^{2 \pi} \int_{0}^{2 \pi} \mathscr{P}^{J K M}(\phi, \vartheta, \psi) d \phi d \psi \\
& =\frac{2 J+1}{2}\left|d_{M K}^{J}(\vartheta)\right|^{2}
\end{aligned}
$$

with $\rho=\cos \vartheta$. This $\rho$-dependent probability function can be expanded as a finite sum of Legendre polynomials ${ }^{21}$

$$
\mathscr{P}^{J K M}(\rho)=\sum_{l=0}^{2 J} c_{l}^{J K M} P_{l}(\rho) .
$$

We use unnormalized Legendre polynomials, i.e., $P_{0}(\rho)=1$, $P_{1}(\rho)=\rho, P_{2}(\rho)=\frac{1}{2}\left(3 \rho^{2}-1\right)$, etc., and

$$
\int_{-1}^{1} P_{l}(\rho) P_{l^{\prime}}(\rho) d \rho=\frac{2}{2 l+1} \delta_{l l^{\prime}} .
$$

The expansion coefficients expressed in $3 j m$ symbols are

$$
\begin{aligned}
c_{l}^{J K M}= & \frac{2 J+1}{2}(-1)^{M=K}(2 l+1)\left(\begin{array}{ccc}
J & J & l \\
M & -M & 0
\end{array}\right) \\
& \times\left(\begin{array}{ccc}
J & J & l \\
K & -K & 0
\end{array}\right) .
\end{aligned}
$$

\section{APPENDIX B}

In this Appendix we will give the proofs for Eqs. (59) and (72) from Sec. IV. We first prove the following theorem.

Theorem 1: For $-1 \leqslant m / j \leqslant 1$ and $-1 \leqslant k / j \leqslant 1$, we have 


$$
\begin{aligned}
I & =\frac{1}{\pi} \int_{\rho_{1}}^{\rho_{2}} \frac{P_{l}(\rho)}{\sqrt{\left(1-2 \frac{m k}{j^{2}} \rho-\left(\frac{m}{j}\right)^{2}-\left(\frac{k}{j}\right)^{2}-\rho^{2}\right)}} d \rho \\
& =P_{l}\left(\frac{m}{j}\right) P_{l}\left(\begin{array}{l}
k \\
j
\end{array}\right),
\end{aligned}
$$

where $\rho_{1}$ and $\rho_{2}$ denote the zero points of the second degree polynomial in $\rho$ under the square root sign, given by

$$
\rho_{1,2}=\frac{m k}{j^{2}} \mp \sqrt{\left[1-\left(\frac{m}{j}\right)^{2}\right]\left[1-\left(\frac{k}{j}\right)^{2}\right]} \text {. }
$$

Substituting,

$$
y=\frac{2 \rho-\rho_{1}-\rho_{2}}{\rho_{2}-\rho_{1}}
$$

into Eq. (B1) and using definitions Eqs. (B2), gives

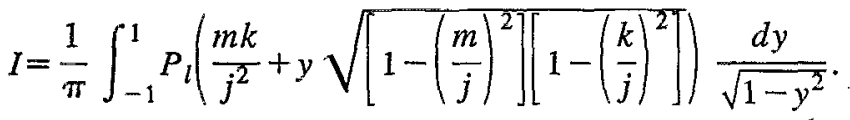

Substituting $-\cos u$ for $y$ gives

$$
I=\frac{1}{\pi} \int_{0}^{\pi} P_{l}\left(\frac{m k}{j^{2}}-\cos u \sqrt{\left[1-\left(\frac{m}{j}\right)^{2}\right]\left[1-\left(\frac{k}{j}\right)^{2}\right]}\right) d u .
$$

We now substitute $m / j=\cos \eta$ and $k / j=\cos \zeta$. Thus we can write the argument of the Legendre polynomial in Eq. (B5) as

$$
\begin{aligned}
\frac{m k}{j^{2}} & -\cos u \sqrt{\left[1-\left(\frac{m}{j}\right)^{2}\right]\left[1-\left(\frac{k}{j}\right)^{2}\right]} \\
& =\cos \eta \cos \zeta-\sin \eta \sin \zeta \cos u=\cos \omega .
\end{aligned}
$$

Physically, we can interpret $\eta$ as the angle between the total angular momentum vector $\mathbf{j}$ and the space-fixed (SF) $z$ axis, $\zeta$ as the angle between $\mathbf{j}$ and the body-fixed (BF) $z$ axis $\left(=z^{\prime}\right)$ in Eq. (12), and $\omega$ as the angle between the SF $z$ axis and the $\mathrm{BF} z$ axis. The angle $u$ can be conceived as the dihedral angle between the plane through $\mathbf{j}$ and the SF $z$ axis and the plane through $\mathbf{j}$ and the BF $z$ axis. We now use the spherical addition theorem, ${ }^{22}$

$$
P_{l}(\cos \omega)=\sum_{q} C_{l q}^{*}(\zeta, 0) C_{l q}(\eta, u),
$$

where $C_{l q}(\eta, u)$ is a spherical harmonic function in the Racah normalization, to rewrite Eq. (B5). Subsequent integration over $u$ only leaves the terms with $q=0$, which themselves are Legendre polynomials, proving Theorem (1).

Using Eqs. (71) and (52) and Theorem (1) and the orthogonality of the Legendre polynomials we can prove Eq. (72). To prove Eq. (59) we multiply Eq. (58) by $P_{l^{\prime}}(\rho)$ and integrate over $\rho$ using, again, Theorem (1) and the orthogonality of the Legendre polynomials.

As an interesting aside we note that one can also derive the expansion coefficients for the classical probability den- sity function (PDF) of Eq. (59) by starting with the quantum mechanical expression of Eq. (A8) and using the correspondence principle, i.e.,

$$
\bar{c}_{l}^{j k m}=\lim _{\hbar \rightarrow 0} c_{l}^{J K M} .
$$

When taking this limit $(j \mathrm{~km})$ and $l$ are fixed and $(J K M)$ is related to $(j \mathrm{~km})$ through Eqs. (54)-(56). Thus taking the limit of $\hbar \rightarrow 0$ corresponds to taking $J \rightarrow \infty$. For $J \gg l$ we have the asymptotic expression for the Clebsch-Gordan (CG) coefficients $^{22-24}$

$$
C_{J M l 0}^{J M} \approx P_{l}\left(\frac{M}{J+1 / 2}\right) .
$$

The $3 \mathrm{jm}$ symbols in Eq. (A8) are related to the CG coefficients by

$$
\left(\begin{array}{ccc}
J & l & J \\
-M & 0 & M
\end{array}\right)=(-1)^{J+M+2 J} \frac{1}{\sqrt{2 J+1}} C_{J K M 0}^{J M}
$$

with, of course, a similar expression for the $3 \mathrm{jm}$ symbol with $K$ instead of $M$ (note that we used the fact that cyclic permutations of the columns leave $3 j m$ symbols unchanged). Furthermore, for large $J$ we have $M /(J+1 / 2) \approx m / j$ and we find

$$
\lim _{\hbar \rightarrow 0} c_{l}^{J K M}=\frac{2 l+1}{2} P_{l}\left(\frac{k}{j}\right) P_{l}\left(\frac{m}{j}\right),
$$

which again is a proof of Eq. (59).

Note, incidentally, that although the Legendre moments of the quantum mechanical PDF converge to the classical Legendre moments for large $J$, there is no pointwise convergence of the quantum mechanical PDF and the classical PDF. In particular, for larger and larger $J_{S}$ the quantum mechanical PDF has an increasing number of zeros. Mathematically, this lack of pointwise convergence is caused by the fact that Eq. (B9) no longer holds when $l$ approaches $J$.

${ }^{1}$ R. B. Bernstein, D. R. Herschbach, and R. D. Levine, J. Phys. Chem. 91, 5365 (1987).

${ }^{2}$ Bernstein Memorial Issue on Molecular Dynamics, J. Phys. Chem. 95, 7961 (1991).

${ }^{3}$ K. H. Kramer and R. B. Bernstein, J. Chem. Phys. 42, 767 (1965).

${ }^{4}$ P. R. Brooks and E. M. Jones, J. Chem. Phys. 45, 3449 (1966).

${ }^{5}$ R. J. Beuhler, Jr., R. B. Bernstein, and K. H. Kramer, J. Am. Chem. Soc. 88, 5331 (1966).

${ }^{6}$ M. H. M. Janssen, D. H. Parker, and S. Stolte, J. Phys. Chem. 95, 8142 (1991).

${ }^{7}$ R. D. Levine and R. B. Bernstein, Chem. Phys. Lett. 105, 467 (1984).

${ }^{8}$ I. W. M. Smith, J. Chem. Educ. 59, 9 (1982).

${ }^{9}$ E. Pollak and R. E. Wyatt, J. Chem. Phys. 78, 4464 (1983).

${ }^{10} \mathrm{H}$. Jalink, G. Nicolasen, D. H. Parker, and S. Stolte (in preparation).

${ }^{11}$ M. H. M. Janssen, D. H. Parker, and S. Stolte, J. Chem. Soc. Faraday Trans. 2 85, 1263 (1989).

${ }^{12}$ A. J. H. M. Meijer, G. C. Groenenboom, and A. van der Avoird, J. Chem. Phys. 101, 7603 (1994).

${ }^{13}$ R. D. Levine and R. B. Bernstein, Molecular Reaction Dynamics and Chemical Reactivity (Oxford University, New York, 1987).

${ }^{14}$ N. C. Blais, R. B. Bernstein, and R. D. Levine, J. Phys. Chem. 89, 10 (1985).

${ }^{15}$ M. H. M. Janssen and S. Stolte, J. Phys. Chem. 91, 5480 (1987).

${ }^{16}$ I. Schechter and R. D. Levíne, J. Chem. Soc. Faraday Trans. 2 85, 1059 (1989). 
${ }^{17}$ S. Stolte, K. K. Chakravorty, R. B. Bernstein, and D. H. Parker, Chem. Phys. 71, 353 (1982).

${ }^{18}$ H. J. Loesch and F. Stienkemeier, J. Chem. Phys. 100, 4308 (1994).

${ }^{19} \mathrm{H}$. Goldstein, Classical Mechanics, 2nd ed. (Addison-Wesley, Reading, 1980).

${ }^{20}$ The NAG Fortran Library Manual, Mark 15 (The Numerical Algorithms Group Limited, Oxford, UK, 1991).
${ }^{21}$ S. E. Choi and R. B. Bernstein, J. Chem. Phys. 85, 150 (1986).

${ }^{22}$ D. M. Brink and G. R. Satchler, Angular Momentum, 2nd ed. (Clarendon, Oxford, 1968)

${ }^{23}$ A. R. Edmonds, Angular Momentum in Quantum Mechanics, 2nd ed. (Princeton, University, New Jersey, 1960).

${ }^{24}$ D. A. Varshalovich, A. N. Moskalev, and V. K. Khersonskii, Quantum Theory of Angular Momentum (World Scientific, Singapore, 1988). 